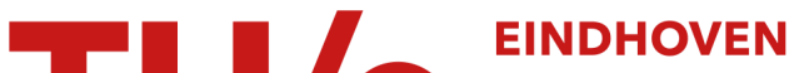 \\ UNIVERSITY OF \\ TECHNOLOGY
}

\section{Performance analysis of tandem queues with small buffers}

Citation for published version (APA):

Vuuren, van, M., \& Adan, I. J. B. F. (2006). Performance analysis of tandem queues with small buffers. (SPORReport : reports in statistics, probability and operations research; Vol. 200612). Technische Universiteit Eindhoven.

Document status and date:

Published: 01/01/2006

\section{Document Version:}

Publisher's PDF, also known as Version of Record (includes final page, issue and volume numbers)

\section{Please check the document version of this publication:}

- A submitted manuscript is the version of the article upon submission and before peer-review. There can be important differences between the submitted version and the official published version of record. People interested in the research are advised to contact the author for the final version of the publication, or visit the $\mathrm{DOI}$ to the publisher's website.

- The final author version and the galley proof are versions of the publication after peer review.

- The final published version features the final layout of the paper including the volume, issue and page numbers.

Link to publication

\section{General rights}

Copyright and moral rights for the publications made accessible in the public portal are retained by the authors and/or other copyright owners and it is a condition of accessing publications that users recognise and abide by the legal requirements associated with these rights.

- Users may download and print one copy of any publication from the public portal for the purpose of private study or research.

- You may not further distribute the material or use it for any profit-making activity or commercial gain

- You may freely distribute the URL identifying the publication in the public portal.

If the publication is distributed under the terms of Article $25 \mathrm{fa}$ of the Dutch Copyright Act, indicated by the "Taverne" license above, please follow below link for the End User Agreement:

www.tue.nl/taverne

Take down policy

If you believe that this document breaches copyright please contact us at:

openaccess@tue.nl

providing details and we will investigate your claim. 


\title{
PERFORMANCE ANALYSIS OF TANDEM QUEUES WITH SMALL BUFFERS
}

\author{
Marcel van Vuuren and Ivo J.B.F. Adan \\ Eindhoven University of Technology, P.O. Box 513, 5600 MB, Eindhoven, The Netherlands \\ E-mail:m.v.vuuren@tue.nl,i.j.b.f.adan@tue.nl
}

\begin{abstract}
In this paper we present an approximation for the performance analysis of single-server tandem queues with small buffers and generally distributed service times. The approximation is based on decomposition of the tandem queue in subsystems, the parameters of which are determined by an iterative algorithm. By employing a detailed description of the service process of each subsystem, we obtain an accurate approximation of performance characteristics such as throughput and mean sojourn time, which substantially outperforms former methods.
\end{abstract}

Keywords: tandem queues, approximation, decomposition, small finite buffers, blocking, production lines.

\section{Introduction}

Queueing networks with finite buffers have been studied extensively in the literature; see, e.g., [2, 7, 9]. These models have many applications in manufacturing, communication and computer systems. In most cases queueing networks with finite buffers are intractable; therefore the majority of the literature is devoted to approximations of performance characteristics such as throughput and mean sojourn times. To the best of our knowledge, the average errors in throughput and mean sojourn time approximations reported in the literature are usually around $5 \%$. Typically, the errors are large (up to 30\%) for systems with small buffers; see e.g. [12]. But, in manufacturing systems, it is common to have small buffers. Hence, good approximations for such systems are definitely needed. In this paper we propose a method for the approximative analysis of single-server tandem queues with general service times, small finite buffers and Blocking After Service (BAS). We are interested in the queue-length distribution of each buffer; these distributions may be used to determine the throughput and mean sojourn time.

In this paper we consider a tandem queue $(L)$ with $N$ servers in tandem and $N-1$ buffers $B_{i}, i=$ $1, \ldots, N-1$, of size $b_{i}$ in between. The servers are labeled $M_{i}, i=0, \ldots, N-1$. The random variable $S_{i}$ denotes the generic service time of server $M_{i} ; S_{i}$ is generally distributed with rate $\mu_{p, i}$ and coefficient of variation $c_{p, i}$. Each server can serve one customer at a time. Server $M_{0}$ is never starved and we consider the BAS blocking protocol. Figure 1 shows a tandem queue with four servers in tandem.

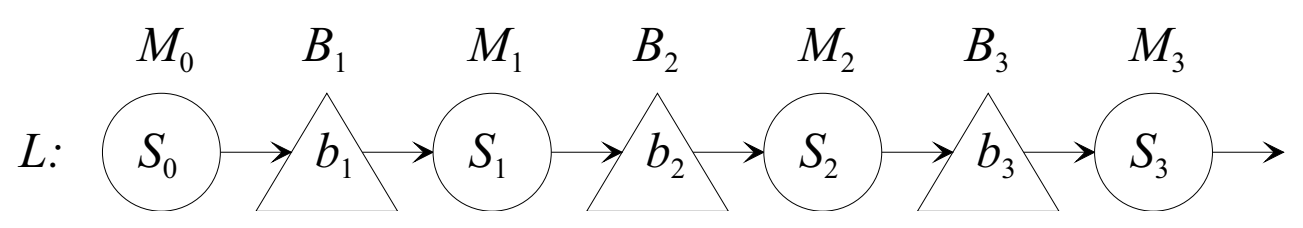

Figure 1: A tandem queue with four servers.

The method to approximate the queue-length distribution of the buffers is based on decomposition of the tandem queue in single-buffer subsystems. Each buffer $B_{i}$ is considered in isolation, where, to take into account the relation with the upstream line and downstream line, the service times of server $M_{i-1}$ in front of buffer $B_{i}$ and server $M_{i}$ after buffer $B_{i}$ are adapted by including possible starvation of $M_{i-1}$ before service and possible blocking of $M_{i}$ after service. It is important to note that dependencies between service time and blocking after service are carefully taken into account; this seems to be the most important source of performance improvement of the proposed approximation. By fitting the first two 
moments, the distributions of the service times including starvation and blocking are approximated by a simple phase-type distributions, the parameters of which are tuned by means of an iterative algorithm.

Decomposition techniques for single-server queueing networks have also been used by a.o. Perros [8], and Kerbache and MacGregor Smith [4]. Van Vuuren et al. [12] extend the decomposition technique to multi-server tandem queues with finite buffers, and show that the quality of the approximation substantially degrades when the buffers become small, i.e., errors can get as large as $30 \%$ in the throughput and mean sojourn time. The results of the current method will be compared with the ones of [12] and also with the ones of the approximation developed by Helber [3], which is the most recent and best method available; the comparison shows that the current method performs substantially better than former methods, with average errors around $1 \%$.

This paper is organized as follows. In Section 2 we explain the decomposition of the tandem queue in subsystems. In the section thereafter we take a closer look at the subsystems. Section 4 describes the iterative algorithm. Numerical results are presented in Section 5 and they are compared with simulation and other approximation methods. Finally, Section 6 contains some concluding remarks.

\section{Decomposition of the tandem queue}

We decompose the original tandem queue $L$ into $N-1$ subsystems $L_{1}, L_{2}, \ldots, L_{N-1}$. Subsystem $L_{i}$ consists of buffer $B_{i}$ of size $b_{i}$, an arrival-server in front of the buffer, and a departure-server after the buffer. In Figure 2 we show the decomposition of line $L$ of Figure 1.

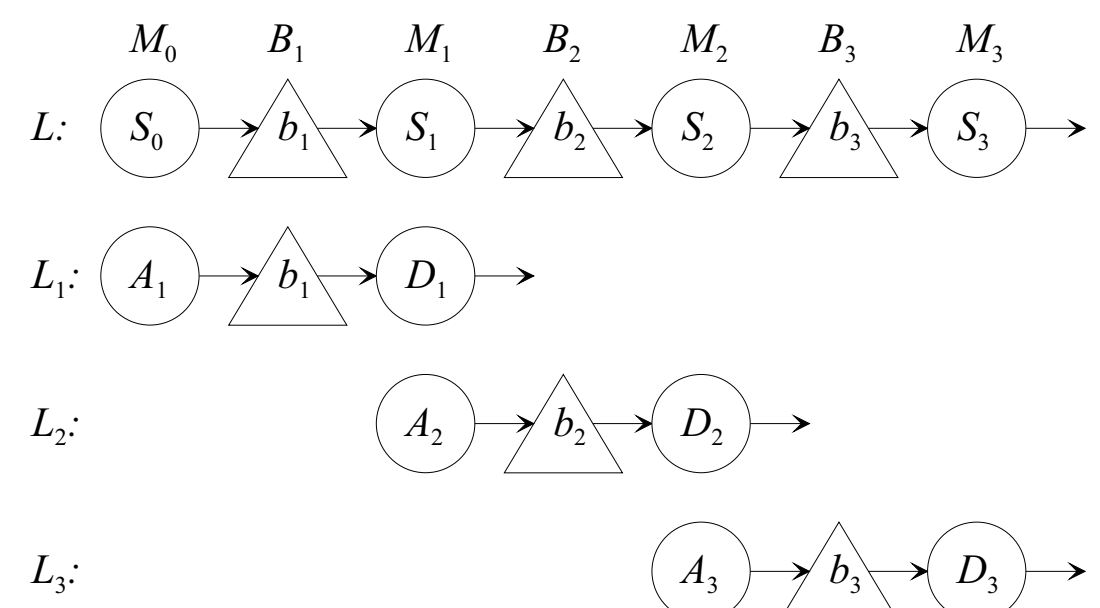

Figure 2: Decomposition of the tandem queue of Figure 1.

The arrival-server of subsystem $L_{i}$ is, of course, server $M_{i-1}$, but, to take into account the connection with the upstream line, its service time is different from $S_{i-1}$. The random variable $A_{i}$ denotes the service time of the arrival-server in subsystem $L_{i}, i=1, \ldots, M-1$. This random variable represents the original service time $S_{i-1}$ of server $M_{i-1}$ including possible starvation of this server before the service start. Similarly, the random variable $D_{i}$ denotes the service time of the departure-server in subsystem $L_{i}$; it represents the service time $S_{i}$ of server $M_{i}$ including possible blocking of this server after service completion.

In the next section we elaborate further on the arrivals at and the departures from the subsystems. 


\section{The subsystems}

In this section we describe how the service times of the arrival and departure server in subsystem $L_{i}$ are modeled. Also, we describe the method to fit the first two moments, the computation of the first two moments of the maximum of two independent phase-type random variables, and the detailed analysis of each subsystem.

\subsection{The arrivals at and the departures from the subsystems}

In the description of the service time $A_{i}$ of arrival-server $M_{i-1}$ of subsystem $L_{i}$ we try to use all information available. Note that an arrival in buffer $B_{i}$, i.e., a customer being served by $M_{i-1}$ moves to buffer $B_{i}$ when space becomes available, corresponds to a departure from the upstream subsystem $L_{i-1}$. Just after this departure, two situations may occur: subsystem $L_{i-1}$ is empty with probability $q_{i-1}^{e}$, or it is not empty with probability $1-q_{i-1}^{e}$ (where, of course, we do not count the customer at arrival-server $M_{i-2}$ as being in $\left.L_{i-1}\right)$. In the former situation, server $M_{i-1}$ has to wait for a residual service time of arrival-server $M_{i-2}$ of subsystem $L_{i-1}$, denoted as $R A_{i-1}$, before the actual service can start. In the latter situation, the actual service $S_{i-1}$ can start immediately. Hence, the service time $A_{i}$ is equal to $R A_{i-1}+S_{i-1}$ with probability $q_{i-1}^{e}$ and equal to $S_{i-1}$ with probability $1-q_{i-1}^{e}$. Figure 3 shows a schematic representation of the service time $A_{i}$.

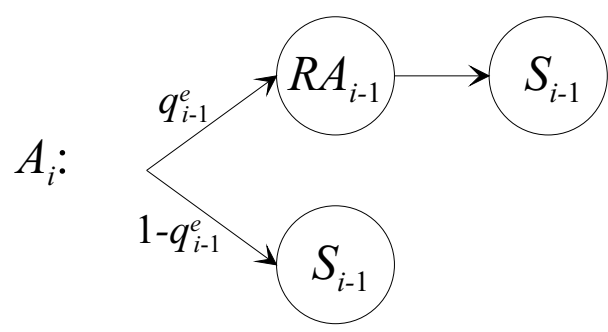

Figure 3: The service time $A_{i}$ of the arrival-server of subsystem $L_{i}$.

We now describe the service time $D_{i}$ of the departure-server $M_{i}$ of subsystem $L_{i}$. Here we distinguish between the service time of a customer for which the previous customer left behind an empty subsystem $L_{i}$ or not; the reason for doing so is that, when time elapses between the departure of the previous customer and the service start of the current customer, it will be less likely that the current one will be blocked. Let the random variable $D_{i}^{e}$ denote the service time of departure-server $M_{i}$ if just after the previous service completion subsystem $L_{i}$ is empty; $D_{i}^{n e}$ is the service time if $L_{i}$ is not empty.

If subsystem $L_{i}$ is empty just after a departure, the next service time starts when a new customer enters subsystem $L_{i}$. Upon arrival of this customer, we have two possibilities: buffer $B_{i+1}$ of the downstream subsystem $L_{i+1}$ is full with probability $p_{i+1}^{f}$, or not. However, this event does not correspond to an arrival or departure in $L_{i+1}$. So $p_{i+1}^{f}$ cannot be calculated as an arrival or departure probability in $L_{i+1}$, but instead, it will be approximated by the equilibrium probability that buffer $B_{i+1}$ is full, given that arrival-server $M_{i}$ in subsystem $L_{i+1}$ is not blocked. If buffer $B_{i+1}$ is full, the service time $D_{i}^{e}$ is the maximum of $S_{i}$ and the residual service time of departure-server $M_{i+1}$ in subsystem $L_{i+1}$; the residual service time of $M_{i+1}$ is approximated by the equilibrium residual service time, denoted as $E D_{i+1}$. If $B_{i+1}$ is not full, the current customer can immediately move to $B_{i+1}$ after completion of $S_{i}$, so then $D_{i}^{e}$ is equal to $S_{i}$. Figure 4 shows a schematic representation of the service time $D_{i}^{e}$.

If subsystem $L_{i}$ is not empty just after a departure, the next service starts immediately. Note that a departure from $L_{i}$ is an arrival at $L_{i+1}$, and on arrival three situations may occur. First, it is possible that the arrival is triggered by a service completion of departure-server $M_{i+1}$ of $L_{i+1}$, i.e., server $M_{i}$ was blocked and waiting for $M_{i+1}$ to complete service. In this situation we say that the status of downstream 


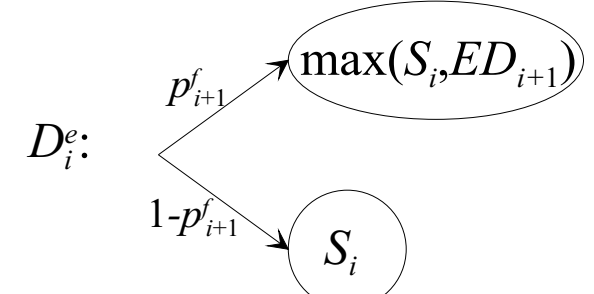

Figure 4: The service time of the departure-server of subsystem $L_{i}$ when the previous customer leaves behind an empty subsystem.

subsystem $L_{i+1}$ changes from blocked to full; the probability that this occurs is denoted by $r_{i+1}^{b f}$. Then the next service of $M_{i}$ and $M_{i+1}$ start simultaneously, so $D_{i}^{n e}$ is equal to the maximum of $S_{i}$ and $D_{i+1}$. Second, just before the arrival there is only one position left in $B_{i+1}$, so right after the arrival $B_{i+1}$ is full; the probability that this happens is denoted by $r_{i+1}^{e f}$. Then, on arrival, departure-server $M_{i+1}$ is already busy with a residual service time $R D_{i+1}$, and thus $D_{i}^{n e}$ is equal to the maximum of $S_{i}$ and $R D_{i+1}$. Finally, when neither of these two situations occurs, server $M_{i}$ can start servicing and after service completion, the customer can immediately move to buffer $B_{i+1}$. Hence, in this case, $D_{i}^{n e}$ is equal to $S_{i}$. Figure 5 shows a schematic representation of the service time $D_{i}^{n e}$.

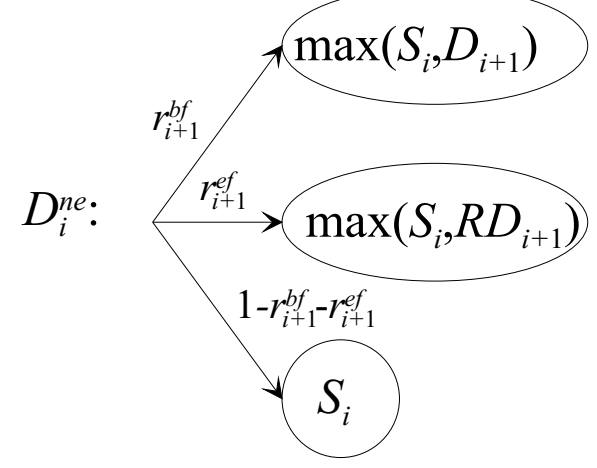

Figure 5: The service time of the departure-server of subsystem $L_{i}$ when the previous customer does not leave behind an empty subsystem.

Remark: There is one exception to the representation of $D_{i}^{n e}$, namely when $b_{i+1}=0$. In the second situation, just before the arrival, server $M_{i+1}$ is available and thus, right after the arrival, both servers $M_{i}$ and $M_{i+1}$ start the next service simultaneously. Hence, $D_{i}^{n e}$ is equal to the maximum of $S_{i}$ and $D_{i+1}$ (instead of $R D_{i+1}$ ).

Summarizing, the service time $D_{i}$ of the current customer is equal to $D_{i}^{e}$ if the previously served customer left behind an empty subsystem and otherwise, it is equal to $D_{i}^{n e}$.

We will approximate the distribution of $A_{i}, D_{i}^{e}$ and $D_{i}^{n e}$ by fitting simple phase-type distributions to the first two moments. In particular, we develop an efficient method to compute the first two moments of the maximum of two phase-type random variables; this will be explained in the following sections.

\subsection{Two moment fit}

We will model the distribution of a random variable with rate $\lambda$ and coefficient of variation $c$ as a mixed Erlang distribution if $c^{2} \leq 1$, and otherwise, as a Hyperexponential distribution (see, e.g., [11]).

More specifically, if $1 / k \leq c^{2} \leq 1 /(k-1)$ for some $k=2,3, \ldots$, then the rate and squared coefficient 
of variation of the Erlang ${ }_{k-1, k}$ distribution with density

$$
f(t)=p \mu^{k-1} \frac{t^{k-2}}{(k-2) !} e^{-\mu t}+(1-p) \mu^{k} \frac{t^{k-1}}{(k-1) !} e^{-\mu t}, \quad t \geq 0,
$$

matches with $\lambda$ and $c_{a}^{2}$, provided the parameters $p$ and $\mu$ are chosen as

$$
p=\frac{1}{1+c^{2}}\left[k c^{2}-\left\{k\left(1+c^{2}\right)-k^{2} c^{2}\right\}^{1 / 2}\right], \quad \mu=(k-p) \lambda .
$$

If $c^{2}>1$, the rate and squared coefficient of variation of the Hyper-exponential distribution with density

$$
f(t)=p \mu_{1} e^{-\mu_{1} t}+(1-p) \mu_{2} e^{-\mu_{2} t}, \quad t \geq 0
$$

matches with $\lambda$ and $c^{2}$, provided the parameters $p, \mu_{1}$ and $\mu_{2}$ are chosen as

$$
p=\frac{1}{2}\left(1+\sqrt{\frac{c^{2}-1}{c^{2}+1}}\right), \quad \mu_{1}=2 p \lambda, \quad \mu_{2}=2(1-p) \lambda .
$$

There exist also other parameter choices for fitting these distributions, and other distributions for fitting. In our experience, use of other distributions or parameters does not essentially affect the quality of the approximation.

Both Erlang and Hyper-exponential distribution belong to the family of Coxian distributions. A random variable is said to have a $\operatorname{Coxian}_{k}$ distribution if it has to go through at most $k$ exponential phases, where phase $i$ has rate $\nu_{i}, i=1, \ldots, k$. It starts in phase 1 and after phase $i, i=1, \ldots, k-1$, it enters phase $i+1$ with probability $p_{i}$, whereas it ends with probability $1-p_{i}$; phase $k$ is the last phase, so $p_{k}=0$. Clearly, the Erlang ${ }_{k-1, k}$ distribution is a Coxian $k$ distribution with $\nu_{i}=\mu$ for all $i$ and $p_{i}=1$ for $i=1, \ldots, k-2$ and $p_{k-1}=1-p$; the Hyper-exponential distribution is a Coxian 2 distribution with

$$
\nu_{1}=\mu_{1}, \quad \nu_{2}=\mu_{2}, \quad p_{1}=(1-p) \frac{\mu_{1}-\mu_{2}}{\mu_{1}},
$$

where, without loss of generality, $\mu_{1} \geq \mu_{2}$. The representation of Erlang and Hyper-exponential distributions in terms of Coxians will be convenient for the description of subsystem $L_{i}$ as a finite-state Markov process in Section 3.4.

\subsection{The maximum of two phase-type random variables}

In this section we calculate the first two moments of the maximum of two independent Erlang distributed random variables. Let $E_{i}$ denote an Erlang $k_{i}$ distributed random variable with scale parameter $\mu_{i}, i=$ 1,2 , and assume that $E_{1}$ and $E_{2}$ are independent. The maximum of $E_{1}$ and $E_{2}$ is phase-type distributed, the first (random) number of exponential phase have rate $\mu_{1}+\mu_{2}$. These phases are followed by a (random) number of exponential phases with rate $\mu_{1}$ or rate $\mu_{2}$, depending on which of the random variables $E_{1}$ and $E_{2}$ finishes first. Let $q_{1, j}$ with $0 \leq j \leq k_{2}-1$ be the probability that $E_{2}$ has completed $j$ phases when $E_{1}$ completes its final phase, and similarly, let $q_{2, i}$ with $0 \leq i \leq k_{1}-1$ be the probability that $E_{1}$ has completed $i$ phases when $E_{2}$ completes its final phase. It is easily verified that $q_{1, j}$ and $q_{2, i}$ both follow a Negative Binomial distribution, i.e.,

$$
\begin{aligned}
q_{1, j} & =\left(\begin{array}{c}
k_{1}-1+j \\
k_{1}-1
\end{array}\right)\left(\frac{\mu_{2}}{\mu_{1}+\mu_{2}}\right)^{j}\left(\frac{\mu_{1}}{\mu_{1}+\mu_{2}}\right)^{k_{1}}, \quad 0 \leq j \leq k_{2}-1, \\
q_{2, i} & =\left(\begin{array}{c}
k_{2}-1+i \\
k_{2}-1
\end{array}\right)\left(\frac{\mu_{1}}{\mu_{1}+\mu_{2}}\right)^{i}\left(\frac{\mu_{2}}{\mu_{1}+\mu_{2}}\right)^{k_{2}}, \quad 0 \leq i \leq k_{1}-1 .
\end{aligned}
$$


Conditioned on the event that $E_{1}$ finishes first and $E_{2}$ has then completed $j$ phases, the maximum of $E_{1}$ and $E_{2}$ is Erlang distributed with $k_{1}+k_{2}$ phases, the first $k_{1}+j$ of which have rate $\mu_{1}+\mu_{2}$ and the last $k_{2}-j$ have rate $\mu_{2}$. Let $M_{1, j}$ denote this conditional maximum, then

$$
\begin{aligned}
& \mathbb{E} M_{1, j}=\frac{k_{1}+j}{\mu_{1}+\mu_{2}}+\frac{k_{2}-j}{\mu_{2}}, \\
& \mathbb{E} M_{1, j}^{2}=\frac{\left(k_{1}+j\right)\left(k_{1}+j+1\right)}{\left(\mu_{1}+\mu_{2}\right)^{2}}+\frac{\left(k_{1}+j\right)\left(k_{2}-j\right)}{\left(\mu_{1}+\mu_{2}\right) \mu_{2}}+\frac{\left(k_{2}-j\right)\left(k_{2}-j+1\right)}{\mu_{2}^{2}} .
\end{aligned}
$$

Similarly, let $M_{2, i}$ denote the maximum of $E_{1}$ and $E_{2}$, conditioned on the event that $E_{2}$ finishes first and $E_{1}$ has then completed $i$ phases. For the first two moments of $M_{2, i}$ we have

$$
\begin{aligned}
& \mathbb{E} M_{2, i}=\frac{k_{2}+i}{\mu_{1}+\mu_{2}}+\frac{k_{1}-i}{\mu_{1}}, \\
& \mathbb{E} M_{2, i}^{2}=\frac{\left(k_{1}+j\right)\left(k_{1}+j+1\right)}{\left(\mu_{1}+\mu_{2}\right)^{2}}+\frac{\left(k_{1}+j\right)\left(k_{2}-j\right)}{\left(\mu_{1}+\mu_{2}\right) \mu_{2}}+\frac{\left(k_{2}-j\right)\left(k_{2}-j+1\right)}{\mu_{2}^{2}} .
\end{aligned}
$$

The first two moments of the maximum of $E_{1}$ and $E_{2}$ can now easily be computed by conditioning on the above events, yielding

$$
\begin{aligned}
\mathbb{E}\left(\max \left\{E_{1}, E_{2}\right\}\right) & =\sum_{j=0}^{k_{2}-1} q_{1, j} \mathbb{E} M_{1, j}+\sum_{i=0}^{k_{1}-1} q_{2, i} \mathbb{E} M_{2, i}, \\
\mathbb{E}\left(\max \left\{E_{1}, E_{2}\right\}^{2}\right) & =\sum_{j=0}^{k_{2}-1} q_{1, j} \mathbb{E} M_{1, j}^{2}+\sum_{i=0}^{k_{1}-1} q_{2, i} \mathbb{E} M_{2, i}^{2} .
\end{aligned}
$$

Note that, if $E_{1}$ and $E_{2}$ are both probabilistic mixtures of Erlang random variables, then the first two moments of the maximum of $E_{1}$ and $E_{2}$ can be computed from the above equations by conditioning on the composition of the maximum.

\subsection{Analyzing a subsystem}

In this section we describe the steady-state analysis of subsystem $L_{i}$; in the remainder of this section we drop the subscript $i$ in the notations. By fitting phase-type distributions on the service times $A$, $D^{e}, D^{n e}$, the subsystem can be described by a finite state Quasi-Birth-and-Death process (QBD), with states $(i, j, k)$. The state variable $i$ denotes the total number of customers in the subsystem. Clearly, $0 \leq i \leq b+2$ where $i=b+2$ indicates that the arrival-server is blocked. The state variable $j(k)$ indicates the phase of the service time of the arrival(departure)-server. The possible values of $j$ are the phases of $A$; the possible values of $k$ are the phases of $D^{e}$ plus the phases of $D^{n e}$.

To define the generator of the QBD we use the Kronecker product: If $A$ is an $n_{1} \times n_{2}$ matrix and $B$ is an $n_{3} \times n_{4}$ matrix, the Kronecker product $A \otimes B$ is defined by

$$
A \otimes B=\left(\begin{array}{ccc}
A(1,1) B & \cdots & A\left(1, n_{2}\right) B \\
\vdots & & \vdots \\
A\left(n_{1}, 1\right) B & \cdots & A\left(n_{1}, n_{2}\right) B
\end{array}\right)
$$

To specify the generator $\mathbf{Q}$ of the QBD, we order the states lexicographically and partition the state space into levels, where level $=0,1, \ldots, b+2$ is the set of all states with $i$ customers in the system. Then $\mathbf{Q}$ 
has the form:

$$
\mathbf{Q}=\left(\begin{array}{cccccc}
B_{00} & B_{01} & & & & \\
B_{10} & A_{1} & A_{0} & & & \\
& A_{2} & \ddots & \ddots & & \\
& & \ddots & \ddots & A_{0} & \\
& & & A_{2} & A_{1} & C_{10} \\
& & & & C_{01} & C_{00}
\end{array}\right)
$$

Below we specify the submatrices in $\mathbf{Q}$. To describe the service process of the arrival and departure server we use the concept of a Markovian Arrival Process (MAP); see [1]. In general, a MAP is defined in terms of a continuous-time Markov process with finite state space $\{0, \cdots, m-1\}$ and generator $G_{0}+G_{1}$. The element $G_{1}(i, j)$ denotes the intensity of transitions from $i$ to $j$ accompanied by an arrival, whereas for $i \neq j$ element $G_{0}(i, j)$ denotes the intensity of the remaining transitions from $i$ to $j$ and the diagonal elements $G_{0}(i, i)$ are negative and chosen such that the row sums of $G_{0}+G_{1}$ are zero.

The MAP for the service process of the departure-server can be described as follows. Assume that the distribution of $D^{n e}$ can be represented by a Coxian distribution with $n_{n e}$ phases, numbered $1, \ldots, n_{n e}$; the starting phase is 1 , the rate of phase $i$ is $\mu_{i}$ and $q_{i}$ is the probability to proceed to the next phase $i+1$, and $1-q_{i}$ is the probability that $D^{n e}$ is finished. Of course, since $n_{n e}$ is the last phase, $q_{n e}=0$. Similarly, the distribution of $D^{e}$ can be represented by a Coxian distribution with $n_{e}$ phases; the starting phase is 1 , the rates are $\nu_{i}$ and the transition probabilities $p_{i}$. Then the states of the MAP are numbered $1, \ldots, n_{n e}+n_{e}$; the first $n_{n e}$ states correspond to the phases of $D^{n e}$ and the last $n_{e}$ states to $D^{e}$. The generator can be expressed as $D E_{0}+D E_{1}$, where the transitions in $D E_{1}$ correspond to departures. So, in $\mathbf{Q}$, the transitions in $D E_{1}$ lead to a transition from level $i$ to $i-1$, whereas the ones in $D E_{0}$ correspond to transitions within level $i$. The non-zero elements of $D E_{0}$ and $D E_{1}$ are specified below.

$$
\begin{aligned}
D E_{0}(i, i) & =-\mu_{i}, & & i=1, \ldots, n_{n e}, \\
D E_{0}(i, i+1) & =q_{i} \mu_{i}, & & i=1, \ldots, n_{n e}-1, \\
D E_{1}(i, 1) & =\left(1-q_{i}\right) \mu_{i}, & & i=1, \ldots, n_{n e}, \\
D E_{0}(i, i) & =-\nu_{i}, & & i=n_{n e}+1, \ldots, n_{n e}+n_{e}, \\
D E_{0}(i, i+1) & =p_{i} \nu_{i}, & & i=n_{n e}+1, \ldots, n_{n e}+n_{e}-1, \\
D E_{1}(i, 1) & =\left(1-p_{i}\right) \nu_{i}, & & i=n_{n e}+1, \ldots, n_{n e}+n_{e} .
\end{aligned}
$$

The MAP for the service process of the arrival-server can be described similarly. Its generator can be expressed as $A R_{0}+A R_{1}$, where the transition rates in $A R_{1}$ are the ones corresponding to a service completion, i.e., an arrival in the buffer. The size of the square matrices $R A_{0}$ and $R A_{1}$ is $n_{a}$ (which is equal to the number of phases of $A$ ). Hence, in $\mathbf{Q}$, the transitions in $A R_{1}$ lead to a transition from level $i$ to $i+1$, whereas the ones in $A R_{0}$ correspond to transitions within level $i$.

Now we can describe the submatrices in $\mathbf{Q}$. The transition rates from levels $1 \leq i \leq b$ are given by

$$
\begin{aligned}
& A_{0}=A R_{1} \otimes I_{n_{n e}+n_{e}}, \\
& A_{1}=A R_{0} \otimes I_{n_{n e}+n_{e}}+I_{n_{a}} \otimes D E_{0}, \\
& A_{2}=I_{n_{a}} \otimes D E_{1},
\end{aligned}
$$

where $I_{n}$ is the identity matrix of size $n$.

In level 0 and level $b+2$ the transition rates are different, because at level 0 the departure-server is idle and at level $b+2$ the arrival-server is blocked. Also, jumps from level 0 to 1 should be directed to the starting phase of $D^{e}$ (instead of the starting phase of $D^{n e}$ ). Hence, 


$$
\begin{aligned}
B_{01} & =A R_{1} \otimes I_{n_{n e}+n_{s_{e}}}\left(n_{n e}+1,:\right) \\
B_{00} & =A R_{0} \\
B_{10} & =I_{n_{a}} \otimes D E_{1}(:, 1) \\
C_{10} & =A R_{1}(:, 1) \otimes I_{n_{n e}+n_{s_{e}}} \\
C_{00} & =D E_{0} \\
C_{01} & =I_{n_{a}}(1,:) \otimes D E_{1}
\end{aligned}
$$

where $X(:, y)$ is the $y$-th column of matrix $X$ and $X(z,:)$ is the $z$-th row of $X$.

This completes the description of the QBD. The steady-state distribution can be determined by the matrix-geometric method. More specifically, we use the efficient techniques developed by Latouche and Ramaswami [5], and Naoumov et al. [10]. If we denote the equilibrium probability vector of level $i$ by $\pi_{i}$, then $\pi_{i}$ has a matrix-geometric form

$$
\pi_{i}=x_{1} R^{i-1}+x_{b+1} \hat{R}^{b+1-i}, \quad i=1, \ldots, b+1,
$$

where $R$ is the minimal nonnegative solution of the matrix-quadratic equation

$$
A_{0}+R A_{1}+R^{2} A_{2}=0,
$$

and $\hat{R}$ is the minimal nonnegative solution of

$$
A_{2}+\hat{R} A_{1}+\hat{R}^{2} A_{0}=0 \text {. }
$$

The matrices $R$ and $\hat{R}$ can be determined by using an iterative algorithm developed by Naoumov et al. [10]. The algorithm for $R$ is listed in Figure 6 .

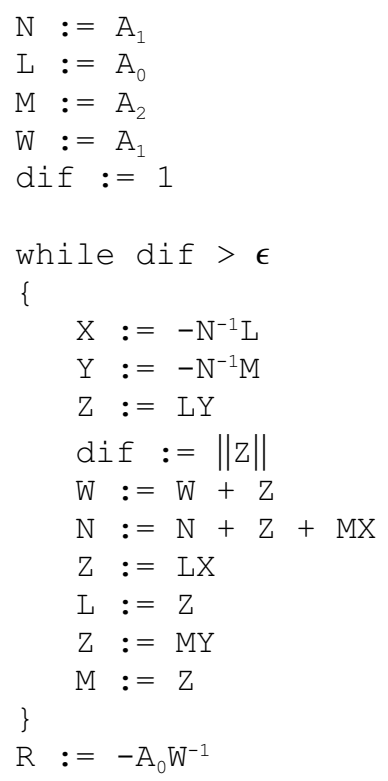

Figure 6: Algorithm of Naoumov et al. [10] to determine the rate matrix $R$, where $\|$.$\| denotes a matrix-$ norm and $\epsilon$ some small positive number. 
The final step is to determine $x_{1}$ and $x_{b+1}$. The balance equations at the boundary levels $0,1, b+1$ and $b+2$ are given by

$$
\begin{aligned}
& 0=\pi_{0} B_{00}+\pi_{1} B_{10}, \\
& 0=\pi_{0} B_{01}+\pi_{1} A_{1}+\pi_{2} A_{2}, \\
& 0=\pi_{b} A_{0}+\pi_{b+1} A_{1}+\pi_{b+2} C_{01}, \\
& 0=\pi_{b} C_{10}+\pi_{b+1} C_{00} .
\end{aligned}
$$

Eliminating $\pi_{0}$ and $\pi_{b+2}$ from the equations above, and then substituting (3) for $\pi_{1}$ and $\pi_{b+1}$ yields

$$
\begin{aligned}
& 0=x_{1}\left(A_{1}+R A_{2}-B_{10} B_{00}^{-1} B_{01}\right)+x_{b+1}\left(\hat{R}^{b} A_{1}+\hat{R}^{b-1} A_{2}-\hat{R}^{b} B_{10} B_{00}^{-1} B_{01}\right), \\
& 0=x_{1}\left(R^{b-1} A_{0}+R^{b} A_{1}-R^{b} C_{10} C_{00}^{-1} C_{01}\right)+x_{b+1}\left(\hat{R} A_{0}+A_{1}-C_{10}\right) C_{00}^{-1} C_{01} .
\end{aligned}
$$

These equations have, together with the normalization equation, a unique solution $x_{1}$ and $x_{b+1}$. Once the queue-length probabilities are known, we can easily derive performance measures and quantities required to describe the service times of the arrival- and departures-server. This is described below.

The Throughput $T$ follows from

$$
T=\pi_{1} B_{10} e+\sum_{i=2}^{b+1} \pi_{i} A_{2} e+\pi_{b+2} C_{01} e,
$$

or equivalently,

$$
T=\pi_{0} B_{01} e+\sum_{i=1}^{b} \pi_{i} A_{0} e+\pi_{b+1} C_{10} e,
$$

where $e$ is the all-one vector.

The probability $q^{e}$ that the subsystem is empty just after a departure, is equal to the mean number of departures per time unit leaving behind an empty system divided by the mean total number of departures per time unit; so

$$
q^{e}=\pi_{1} B_{10} e / T .
$$

The moments of $R A$ can be computed straight-forwardly, once the phase of the service time of the arrival-server, just after a departure leaving behind an empty system, is known. To determine the distribution of the service phase, note that component $(j, k)$ of the vector $\pi_{1} B_{10}$ gives the mean number of departures per time unit from state $(j, k)$ at level 1 . By adding all components with $j=i$ and dividing by $\pi_{1} B_{10} e$, we obtain the probability that the arrival-server is in phase $i$ just after a departure leaving behind an empty system.

The probability $p^{f}$ that the buffer is full, given that the arrival-server is not blocked, satisfies

$$
p^{f}=\frac{\pi_{b+1} e}{\sum_{i=0}^{b+1} \pi_{i} e} .
$$

The moments of $E D$ can be calculated once the phase of the service time of the departure-server is known. To obtain the distribution of the service phase, note that component $(j, k)$ of vector $\pi_{b+1}$ is the 
probability that the arrival-server is in phase $j$, the departure-server is in phase $k$ and the buffer is full. Hence, addition of all components of $\pi_{b+1}$ with $k=i$ and division by $\pi_{b+1} e$ yields the probability that the departure-server is in phase $i$, given that the buffer is full and arrival-server is not blocked.

The probabilities $r^{b f}$ and $r^{e f}$ follow from

$$
r^{b f}=\pi_{b+2} C_{01} e / T, \quad r^{e f}=\pi_{b} A_{0} e / T .
$$

The moments of $R D$, finally, require the phase of the service time of the departure-server just after an arrival saturating the buffer. To find the distribution of the service phase, note that component $(j, k)$ of $\pi_{b} A_{0}$ is equal to the mean number of arrivals per time unit from state $(j, k)$ at level $b$. So, by adding over all components with $k=i$ and dividing by $\pi_{b} A_{0} e$, yields the probability that the departure-server is in phase $i$, just after an arrival saturating the buffer.

\section{The iterative algorithm}

Now we describe the iterative algorithm to approximate the performance of tandem queue $L$. The algorithm is based on decomposition of $L$ in $N-1$ subsystems $L_{1}, L_{2}, \ldots, L_{N-1}$. Before going into detail in Section 4.2, we present the outline of the algorithm in Section 4.1.

\subsection{Outline of the algorithm}

- Step 0: Choose initial characteristics of the service times of the departure-servers for all subsystems $L_{1}, \ldots, L_{N-1}$.

- Step 1: For subsystem $L_{i}=L_{1}, \ldots, L_{N-1}$ :

1. Determine the first two moments of the service time $A_{i}$ of the arrival-server, given the queuelength distribution and throughput of subsystem $L_{i-1}$.

2. Determine the queue-length distribution of subsystem $L_{i}$.

3. Determine the throughput $T_{i}$ of subsystem $L_{i}$.

- Step 2: Determine the new characteristics of the service times of the departure-servers for all subsystems $L_{N-1}, \ldots, L_{1}$.

- Repeat Step 1 and 2 until the characteristics of the service times of the departure-servers have converged.

\subsection{Details of the algorithm}

\section{Step 0: Initialization}

The first step of the algorithm is to initially assume that there is no blocking. This means that the random variables $D_{i}^{e}$ and $D_{i}^{n e}$ are initially the same as the service times $S_{i}$.

\section{Step 1: Evaluation of subsystems}

We know the first two moments of $D_{i}^{e}$ and $D_{i}^{n e}$, but we also need to know the first two moments of $A_{i}$, before we are able to determine the queue-length distribution of $L_{i}$.

(a) The arrival process 
For the first subsystem $L_{1}$, the service time $A_{1}$ is equal to $S_{0}$, because server $M_{0}$ cannot be starved.

For the other subsystems we proceed as follows in order to determine the first two moments of $A_{i}$. By Little's law we have for the throughput $T_{i}$ of subsystem $L_{i}$,

$$
T_{i}=\left(1-p_{i, b_{i}+2}\right) \mu_{a, i},
$$

where $p_{i, b_{i}+2}$ denotes the fraction of time the arrival-server of subsystem $L_{i}$ is blocked and $\mu_{a, i}$ is the mean arrival rate at subsystem $L_{i}$, i.e., $\mu_{a, i}=1 / \mathbb{E}\left(A_{i}\right)$. By substituting the estimate $T_{i-1}^{(k)}$ for $T_{i}$, which is the principle of conservation of flow, and $p_{i, b_{i}+2}^{(k-1)}$ for $p_{i, b_{i}+2}$ we get as new estimate for $\mu_{a, i}$,

$$
\mu_{a, i}^{(k)}=\frac{T_{i-1}^{(k)}}{1-p_{i, b_{i}+2}^{(k-1)}},
$$

where the superscripts indicate in which iteration the quantities have been calculated.

The second moment of $A_{i}$ cannot be determined by using Little's law; instead we calculate $q_{i-1}^{e}$ and the first two moments of $R A_{i-1}$ from the queue length distribution of $L_{i-1}$ and then determine the second moment of $A_{i}$ according to the representation in Figure 3.

\section{(b) Analysis of subsystem $L_{i}$}

Based on the (new) estimates for the first two moments of $A_{i}, D_{i}^{e}$ and $D_{i}^{n e}$ we can determine the steadystate queue length distribution of subsystem $L_{i}$. To do so we first fit mixed Erlang or Hyper-exponential distributions on the first two moments of $A_{i}, D_{i}^{e}$ and $D_{i}^{n e}$, as described in Section 3.2. Then we calculate the equilibrium probability vectors $\pi_{i}$ as described in Section 3.4.

\section{(c) Determining the throughput of $L_{i}$}

Once the steady-state distribution is known, we can determine the new throughput $T_{i}^{(k)}$ according to (4) or equivalently,

$$
T_{i}^{(k)}=\left(1-p_{i, 0}^{(k)}\right) \mu_{d, i}^{(k-1)},
$$

where $p_{i, 0}$ denotes the fraction of time subsystem $L_{i}$ is empty, and $\mu_{d, i}$ is the mean departure rate of subsystem $L_{i}$, i.e., $\mu_{d, i}=1 /\left(q_{i}^{e} \mathbb{E}\left(D_{i}^{e}\right)+\left(1-q_{i}^{e}\right) \mathbb{E}\left(D_{i}^{n e}\right)\right)$.

Further we determine new estimates for the probabilities $p_{i}^{f}, r_{i}^{b f}$ and $r_{i}^{e f}$ and the first two moments of $E D_{i}$ and $R D_{i}$, as explained in Section 3.4.

We perform Step 1 for every subsystem from $L_{1}$ up to $L_{N-1}$.

\section{Step 2: The departure process}

Now we have new estimates for the probabilities $p_{i}^{f}, r_{i}^{b f}$ and $r_{i}^{e f}$ and the first two moments of $E D_{i}$ and $R D_{i}$, and hence, we can recalculate the first two moments of $D_{i}^{e}$ and $D_{i}^{n e}$, starting from subsystem $L_{N-2}$ down to $L_{1}$. Note that $D_{N-1}^{e}$ and $D_{N-1}^{n e}$ are always the same as $S_{M-1}$, because server $M_{N-1}$ can never be blocked.

The calculation of the first two moments of $D_{i}^{e}$ and $D_{i}^{n e}$ is based on the representation shown in Figures 4 and 5.

\section{Convergence}

After Step 1 and 2 we can check whether the iterative algorithm has converged or not. We check this by comparing the mean departure rates in the $(k-1)$-th and $k$-th iteration. When the sum of the absolute values of the differences between these rates is less than $\varepsilon$ we stop; otherwise we repeat Step 1 and 2.

Of course, we may use other stop-criteria as well; for example, we may consider the throughput instead 
of the departure rates. Bottom line is that we go on until 'nothing' changes anymore.

\section{Numerical Results}

In this section we investigate the quality of the proposed approximation by comparing it with discrete event simulation. We also compare the results with an approximation of Van Vuuren et al. [12] and an approximation of Helber [3]. The approximation of Van Vuuren et al. [12] was originally developed for multi-server systems, and it appeared to be less accurate for small-buffered single-server tandem queues. The approximation of Helber [3] is the most recent and best available method in the literature; therefore it is worthwhile to compare the present method with this one. Both methods are based on decomposition, but differ in the description of the subsystems and the iterative method.

In order to investigate the quality of the current method we will evaluate a large set of examples; In each example we assume that only mean and the squared coefficient of variation of the service times at each server are known, and we fit mixed Erlang or Hyper-exponential distributions on the first two moments of the service times, depending on whether the coefficient of variation is less or greater than 1 ; see Section 3.2. Then we will compare the throughput and the mean sojourn time (i.e., the mean time that elapses from the service start at server $M_{1}$ until service completion at server $M_{N-1}$ ) produced by the approximation with the ones produced by discrete event simulation. We are especially interested in investigating for which set of input parameters the current method gives satisfying results. Each simulation run is sufficiently long such that the widths of the $95 \%$ confidence intervals of the mean sojourn time and the delay probability are smaller than $1 \%$.

We use a broad set of parameters for the tests. The mean service times of the servers are all 1 . We vary the number of servers in the tandem queue between $4,8,12$ and 16. The squared coefficient of variation $(\mathrm{SCV})$ of the service times of each server is the same and is varied between 0.2, 0.4, 1 and 2. The buffer sizes between the servers are the same and varied between $0,1,3$, and 5 . We can also test three kinds of imbalance in the tandem queue. We test imbalance in the mean service times by increasing the average service time of the 'even' servers form 1 to 1.2. Imbalance in the SCV is tested by increasing the SCV of the service times of the 'even' servers by 0.5 . Finally, imbalance in the buffer sizes is tested by increasing the buffers size of the 'even' buffers by 2 . This leads to a total of $4^{3} 2^{3}=512$ test cases. The results for each category are summarized in Tables 1 up to 4 . Each table lists the average error in the throughput and the mean sojourn time compared with simulation results. Each table also gives for 3 error-ranges the percentage of the cases which fall in that range, the average error of the approximation from Van Vuuren et al.[12], which is denoted by VAR and the average error of the approximation from Helber [3], which is denoted by Hel. Note that the approximation from Helber [3] can only handle cases with squared coefficients of variation of the service times larger than 0.5 .

\begin{tabular}{|c|r|r|r|r|r|r|r|r|r|r|r|r|}
\hline $\begin{array}{c}\text { Buffer } \\
\text { sizes }\end{array}$ & \multicolumn{5}{|c|}{ Error (\%) in the throughput } & \multicolumn{5}{|c|}{ Error (\%) in mean sojourn time } \\
\cline { 2 - 12 } & Avg. & $0-2$ & $2-4$ & $>4$ & VAR & Hel & Avg. & $0-2$ & $2-4$ & $>4$ & VAR & Hel \\
\hline $0,0, \ldots$ & 1.15 & 81.3 & 14.1 & 4.7 & 11.17 & 5.02 & 2.06 & 59.4 & 28.1 & 12.5 & 12.22 & 7.14 \\
$1,1, \ldots$ & 1.21 & 79.7 & 20.3 & 0.0 & 5.64 & 7.40 & 1.24 & 75.0 & 25.0 & 0.0 & 7.03 & 10.54 \\
$3,3, \ldots$ & 1.49 & 70.3 & 29.7 & 0.0 & 2.89 & 6.90 & 1.13 & 79.7 & 20.3 & 0.0 & 3.95 & 11.35 \\
$5,5, \ldots$ & 1.65 & 62.5 & 34.4 & 3.1 & 2.12 & 5.84 & 1.29 & 75.0 & 23.4 & 1.6 & 2.81 & 10.87 \\
$0,2, \ldots$ & 1.18 & 81.3 & 7.8 & 10.9 & 5.54 & 5.77 & 1.19 & 87.5 & 12.5 & 0.0 & 6.92 & 8.71 \\
$1,3, \ldots$ & 1.02 & 87.5 & 12.5 & 0.0 & 3.55 & 6.64 & 0.84 & 93.8 & 6.3 & 0.0 & 5.02 & 10.48 \\
$3,5, \ldots$ & 1.45 & 70.3 & 29.7 & 0.0 & 2.29 & 6.10 & 1.33 & 76.6 & 20.3 & 3.1 & 3.51 & 10.88 \\
$5,7, \ldots$ & 1.60 & 64.1 & 32.8 & 3.1 & 1.84 & 5.28 & 1.76 & 64.1 & 28.1 & 7.8 & 2.87 & 10.56 \\
\hline
\end{tabular}

Table 1: Overall results for tandem queues with different buffer sizes.

Overall we can conclude from the above results that the approximation method works very well. The average error in the throughput is around $1.4 \%$ and the average error in the mean sojourn time is around $1.3 \%$. In most cases the errors are within $1 \%$-width confidence interval of the simulation results. When 


\begin{tabular}{|c|r|r|r|r|r|r|r|r|r|r|r|r|}
\hline \multirow{2}{*}{ SCVs } & \multicolumn{9}{|c|}{ Error (\%) in the throughput } & \multicolumn{5}{|c|}{ Error (\%) in mean sojourn time } \\
\cline { 2 - 13 } & Avg. & $0-2$ & $2-4$ & $>4$ & VAR & Hel & Avg. & $0-2$ & $2-4$ & $>4$ & VAR & Hel \\
\hline $0.2,0.2, \ldots$ & 0.84 & 90.6 & 9.4 & 0.0 & 1.57 & - & 1.71 & 75.0 & 15.6 & 9.4 & 1.58 & - \\
$0.4,0.4, \ldots$ & 0.70 & 92.2 & 7.8 & 0.0 & 0.85 & - & 0.97 & 84.4 & 14.1 & 1.6 & 2.72 & - \\
$1,1, \ldots$ & 0.99 & 87.5 & 12.5 & 0.0 & 3.54 & 3.32 & 0.78 & 92.2 & 7.8 & 0.0 & 5.79 & 8.50 \\
$2,2, \ldots$ & 2.12 & 48.4 & 46.9 & 4.7 & 9.34 & 7.49 & 1.75 & 59.4 & 34.4 & 6.3 & 9.91 & 11.20 \\
$0.2,0.7, \ldots$ & 1.22 & 76.6 & 20.3 & 3.1 & 1.54 & - & 1.48 & 71.9 & 26.6 & 1.6 & 2.97 & - \\
$0.4,0.9, \ldots$ & 1.12 & 81.3 & 18.8 & 0.0 & 2.22 & - & 1.14 & 84.4 & 15.6 & 0.0 & 3.86 & - \\
$1,1.5, \ldots$ & 1.47 & 70.3 & 29.7 & 0.0 & 5.24 & 4.91 & 1.05 & 84.4 & 15.6 & 0.0 & 6.75 & 8.91 \\
$2,2.5, \ldots$ & 2.27 & 50.0 & 35.9 & 14.1 & 10.74 & 8.75 & 1.96 & 59.4 & 34.4 & 6.3 & 10.76 & 11.66 \\
\hline
\end{tabular}

Table 2: Overall results for tandem queues with different SCVs of the service times.

\begin{tabular}{|c|r|r|r|r|r|r|r|r|r|r|r|r|}
\hline \multicolumn{1}{l|}{$\begin{array}{l}\text { Mean } \\
\text { service times }\end{array}$} & \multicolumn{4}{|c|}{ Error (\%) in the throughput } & \multicolumn{4}{|c|}{ Error (\%) in mean sojourn time } \\
\cline { 2 - 24 } & Avg. & $0-2$ & $2-4$ & $>4$ & VAR & Hel & Avg. & $0-2$ & $2-4$ & $>4$ & VAR & Hel \\
\hline $1,1, \ldots$ & 1.07 & 84.0 & 14.1 & 2.0 & 4.06 & 5.67 & 1.12 & 81.3 & 17.2 & 1.6 & 5.10 & 9.74 \\
$1,1.2, \ldots$ & 1.62 & 65.2 & 31.3 & 3.5 & 4.70 & 6.57 & 1.59 & 71.5 & 23.8 & 4.7 & 5.98 & 10.40 \\
\hline
\end{tabular}

Table 3: Overall results for tandem queues with different service rates.

the results of the approximation are compared with those of the VAR and Hel approximations, we see that the new approximation performs substantially better than both other approximations.

Let us look at the results in more detail. If we look at Table 1, we see that the quality of the results for the throughput is nearly insensitive to the buffer sizes. The errors in the mean sojourn time are slightly larger for cases with zero buffers than for cases with non-zero buffers. But the results are still highly acceptable. Another remark is that the approximation does not seem to be very sensitive to imbalance in the buffer sizes.

In Table 2 we see that the error in the throughput is slightly increasing in the SCVs of the service times. For the mean sojourn the times the approximation performs best when the SCVs are around 1. Here, also, the quality of the approximation is not significantly sensitive to imbalance in the SCVs of the service times. On the other hand, Table 3 shows that quality of the approximation slightly depends on imbalance in the mean service times.

Finally in Table 4, it is shown that, as expected, the errors in both the throughput and the mean sojourn time increase when the tandem queue increases in length. This is the case, because an increasingly larger part of the line is described by a single server in the subsystems. But, as the length of the line increases, the results remain highly acceptable.

\section{Concluding remarks}

In this paper we described an algorithm for approximating single-server tandem queues with small buffers. We used a decomposition approach and developed an iterative algorithm to approximate the performance characteristics of the tandem queue. To improve the algorithm over existing ones, we modeled the service processes at the subsystems in more detail, in particular taking into account dependencies between service times and blocking after service. The queue-length distributions of the subsystems are determined by using the matrix-analytic method.

We tested the algorithm by comparing it with a discrete-event simulation and with existing methods and the results are very good. The average errors in both the throughput and the mean sojourn time are around $1.3 \%$, where the average errors in existing methods are between 5 and $10 \%$. Hence, it now seems possible to get reliable approximations for single-server tandem queues with small or zero buffers. 


\begin{tabular}{|r|r|r|r|r|r|r|r|r|r|r|r|r|}
\hline Servers & \multicolumn{5}{|c|}{ Error (\%) in the throughput } & \multicolumn{5}{c|}{ Error (\%) in mean sojourn time } \\
\cline { 2 - 13 } in line & Avg. & $0-2$ & $2-4$ & $>4$ & VAR & Hel & Avg. & $0-2$ & $2-4$ & $>4$ & VAR & Hel \\
\hline 4 & 0.52 & 96.9 & 3.1 & 0.0 & 1.48 & 1.55 & 0.73 & 94.5 & 5.5 & 0.0 & 1.78 & 1.44 \\
8 & 1.04 & 88.3 & 11.7 & 0.0 & 3.91 & 4.91 & 0.98 & 92.2 & 7.0 & 0.8 & 4.71 & 6.47 \\
12 & 1.67 & 64.1 & 32.8 & 3.1 & 5.42 & 7.93 & 1.48 & 74.2 & 21.1 & 4.7 & 6.69 & 13.11 \\
16 & 2.15 & 49.2 & 43.0 & 7.8 & 6.71 & 10.08 & 2.23 & 44.5 & 48.4 & 7.0 & 8.98 & 19.24 \\
\hline
\end{tabular}

Table 4: Overall results for tandem queues of different length.

\section{References}

[1] S. Asmussen and G. Koole (1993) Marked point processes as limits of Markovian arrival streams. Journal of Applied Probability 30, 365-372.

[2] Y. Dallery and B. Gershwin (1992) Manufacturing flow line systems: a review of models and analytical results. Queueing Systems 12, 3-94.

[3] S. Helber (2005) Analysis of Flow Lines with Cox-2-distributed Processing Times and Limited Buffer Capacity OR Spectrum 27, 221-242.

[4] L. Kerbache and J. MacGregor Smith (1987) The Generalized Expansion Method for Open Finite Queueing Networks. The European Journal of Operations Research 32, 448-461.

[5] G. Latouche and V. Ramaswami (1999) Introduction to Matrix Analytic Methods in Stochastic Modeling. ASA-SIAM Series on Statistics and Applied Probability 5.

[6] R.A. Marie (1980) Calculating equilibrium probabilities for $\lambda(n) / C_{k} / 1 / N$ queue. Proceedings Performance '80, Toronto, 117-125.

[7] H.G. Perros (1989) A Bibliography of Papers on Queueing Networks with Finite Capacity Queues. Perf. Eval. 10, 255-260.

[8] H.G. Perros (1994) Queueing Networks with Blocking. Oxford University Press.

[9] H.G. Perros and T. Altiok (1989) Queueing Networks with Blocking North-Holland, Amsterdam.

[10] V. Naoumov, U.R. Krieger, D. Wagner (1997) Analysis of a Multiserver Delay-Loss System with a General Markovian Arrival Process. Matrix-Analytic Methods in Stochastic Models (A.S.Alfa and S.R.Chakravarthy eds), Lecture Notes in Pure and Applied Mathematics, 183, Marcel Dekker, New York, 1996.

[11] H.C. Tijms (1994) Stochastic models: an algorithmic approach. John Wiley \& Sons, Chichester.

[12] M. van Vuuren, I.J.B.F. Adan and S.A. Resing-Sassen (2003) Performance Analysis of Multi-Server Tandem Queues with Finite Buffers and blocking. OR Spectrum 27, . 